\title{
Ruling out a critical density baryonic universe
}

\author{
Michael Birkel and Subir Sarkar* \\ Theoretical Physics, University of Oxford, \\ 1 Keble Road, Oxford OX1 3NP, UK \\ (submitted to Physics Letters B)
}

\begin{abstract}
It has been suggested by Bartlett and Hall that our universe may have the critical density in baryons by virtue of specific interactions with a 'shadow' world. We show that this possibility is severely constrained by primordial nucleosynthesis, stellar evolution and the thermalization of the cosmic microwave background. In particular, recent observations of small angular-scale anisotropy in the cosmic microwave background conclusively rule out all such baryon-dominated cosmologies.
\end{abstract}

98.80.Cq, 97.60.Bw,98.70.Vc,98.80.Ft

*PPARC Advanced Fellow

hep-ph/9705331 


\section{INTRODUCTION}

The 'fine tuning' problems of the Friedmann-Robertson-Walker cosmology favour the possibility that the universe is spatially flat with density parameter $\Omega=1$, as would result naturally from an inflationary phase in the early universe [1]. However, the observed abundance of deuterium, which could only have been formed during big bang nucleosynthesis (BBN) [2], implies an upper bound of $\Omega_{\mathrm{N}}<0.033 h^{-2}$ on the nucleonic contribution to the density parameter [3]. Since the Hubble parameter $h\left(\equiv H_{0} / 100 \mathrm{~km} \mathrm{sec}^{-1} \mathrm{Mpc}^{-1}\right)$ is in the range $\sim 0.5-1$, ordinary matter cannot close the universe and this discrepancy has motivated the much discussed idea that non-baryonic dark matter makes up the difference. This would require the existence of new stable massive particles in extensions of physics beyond the Standard Model, e.g. massive neutrinos or the lightest supersymmetric particle [6]. It is clearly important to establish whether there are any loopholes in this argument, especially since nucleons can in principle make up all the dynamically detected dark matter which amounts to only $\Omega \sim 0.1$ [1.]1. In particular, recent observations of microlensing of stars in the Large Magellanic Cloud indicate that $\sim 0.5 M_{\odot}$ objects (probably old white dwarfs) can already account for upto half of the dark matter in the halo of our Galaxy [8].

There have been several proposals for relaxing the BBN bound on the nucleon density by either modifying general relativity [9], or complicating the standard picture of nucleosynthesis, e.g. by invoking non-zero neutrino chemical potentials [10] or inhomogeneities in the nucleon distribution resulting from a first-order quark-hadron phase transition [11]. The latter possibility is physically the best motivated but detailed studies show that even with new free parameters it is difficult to permit the critical density in nucleons 12]. There have also been suggestions which invoke physics beyond the Standard Model [13]. One requires a tau neutrino with a mass of 20-30 MeV (but with its relic abundance suppressed by a factor of $\sim 10$ below the nominal 'freeze-out' value) decaying 'invisibly' into electron neutrinos with a lifetime of 200-1000 sec 14. Although this would allow a $\Omega_{\mathrm{N}}=1$ universe, the model is rather fine-tuned and quite unlikely from the particle physics viewpoint; in particular the $\nu_{\tau}$ must have its tree-level Standard Model decays $\left(\nu_{\tau} \rightarrow e^{+} e^{-} \nu_{e}\right)$ totally suppressed as these would disrupt nucleosynthesis [15,13]; moreover the required mass region is now almost ruled out experimentally [16]. A better motivated suggestion [17] is that the hadronic and radiation cascades triggered by the decay after BBN of massive unstable particles such as gravitinos can process the elemental yields so as to synthesize adequate amounts of deuterium even for $\Omega_{\mathrm{N}}=1$. However, there is a problem with the abundance of ${ }^{6} \mathrm{Li}$, which, in this model, is nearly as abundant as ${ }^{7} \mathrm{Li}$, in apparent conflict with observations 18 . (The same comment applies to the model invoking Hawking radiation from primordial black holes [19].)

In this paper we consider another exotic idea due to Bartlett and Hall [20] viz. that our universe (the 'visible' sector) may have become coupled to a 'hidden' sector through a phase transition after nucleosynthesis. The mixing of photons in the visible sector with their counterpart 'paraphotons' in the hidden sector can then result in a sudden decrease of the comoving photon density. Thus the universe may well have the critical density in nucleons today, while having a nucleon-to-photon ratio during $\mathrm{BBN}$ consistent with observed elemental abundances. To see this we note that the

\footnotetext{
${ }^{1}$ There may exist new forms of baryonic matter which do not participate in nucleosynthesis, e.g. strange quark matter 'nuggets' 河. If these survived baryon evaporation, they could make a substantial contribution to $\Omega$ today [5]; therefore we distinguish between baryons and nucleons in the context of BBN.
} 
nucleon mass density in ratio to the critical density is

$$
\Omega_{\mathrm{N}}=\frac{m_{\mathrm{N}} n_{\mathrm{N}}}{\rho_{\mathrm{c}}}=\frac{8 \pi m_{\mathrm{N}}}{3 H_{0}^{2} M_{\mathrm{P}}^{2}}\left(\frac{n_{\mathrm{N}}}{n_{\gamma}}\right)_{0} \frac{2 \zeta(3) T_{0}^{3}}{\pi^{2}},
$$

where the present temperature of the blackbody cosmic microwave background $(\mathrm{CMB})$ is $T_{0}=$ $2.728 \pm 0.004 \mathrm{~K}(95 \%$ c.l. $)$ [21]. Therefore we require

$$
\left.\eta_{\mathrm{c}} \equiv \frac{n_{\mathrm{N}}}{n_{\gamma}}\right|_{\Omega_{\mathrm{N}}=1} \simeq 2.72 \times 10^{-8} h^{2} \Theta^{-3},
$$

for a critical density in nucleons, where $\Theta \equiv T_{0} / 2.73 \mathrm{~K}$. In the standard cosmology, the comoving entropy does not change after $e^{+} e^{-}$annihilation so the nucleon-to-photon ratio during nucleosynthesis equals its value today, i.e. $\eta_{\mathrm{BBN}}=\eta_{0}$. As mentioned earlier, the observational lower limit to the abundance of deuterium (together with observational upper limits to the abundances of helium and lithium) requires $\eta_{\mathrm{BBN}}<8.9 \times 10^{-10}$ [3]. The interesting possibility suggested in ref. [20] is that the comoving photon number decreases following nucleosynthesis, so that $\eta_{0}$ is raised to $\sim(10-100) \eta_{\mathrm{BBN}}$ and can be as high as $\eta_{\mathrm{c}}$.

We examine the effects of such physics on various cosmological and astrophysical processes such as BBN itself, the spectrum of the CMB and stellar evolution. It turns out that these arguments severely constrain but cannot quite rule out the scenario. However, all models in which the critical density is comprised entirely by baryons (nucleonic or otherwise) are shown to be definitively excluded by recent measurements of anisotropy in the CMB together with measurements of the power spectrum of large-scale structure (LSS) in the universe. Thus, there appears to be no alternative to non-baryonic dark matter if indeed $\Omega \gtrsim 0.1$.

\section{THE PHOTON COOLING COSMOLOGY}

First we briefly review the scenario of Bartlett and Hall [20]. They consider a gauge theory with the group $G \otimes G^{\prime}$ at energies far above the electroweak scale ( $v \simeq 246 \mathrm{GeV}$ ). The group $G$ contains the Standard $S U(3) \otimes S U(2) \otimes U(1)$ Model while $G^{\prime}$ corresponds to a hidden sector with no tree-level renormalizable couplings to known particles although similar in its symmetry breaking pattern. In particular $G^{\prime}$ breaks to a group which includes a hidden (unbroken) electromagnetism $U(1)^{\prime}$ at a scale $v^{\prime} \ll v$ and the $\mathcal{O}(100)$ light degrees of freedom in $G^{\prime}$ (generically termed $X^{\prime}$ ) acquire a mass at this scale. The cosmology is thus quite novel. The lightest 'connector' particle (carrying both $G$ and $G^{\prime}$ quantum numbers) has a mass $M_{\mathrm{C}} \gg v$ so that scattering processes involving the connector particles maintain the two sectors in thermal equilibrium at $T \gg M_{\mathrm{C}}$.

At lower temperatures these reactions freeze-out and the two sectors decouple. Subsequent phase transitions and annihilations of heavy species affect the sectors differently resulting in $T^{\prime}<T$. (For example if the temperature difference is dominantly due to heavy particle annihilations in the visible sector then $T / T^{\prime} \simeq 2(11 / 4)^{1 / 3} \simeq 3$ at $v^{\prime} \ll T \ll m_{e}[20]$.) Thus, during BBN the universe contains two plasmas which are not in thermal contact with each other. At some point after nucleosynthesis a 'recoupling' reaction reestablishes thermal contact, cooling the photons in the visible sector and hence reducing the comoving photon number density. Such a reaction requires a renormalizable interaction between photons and the $X^{\prime}$ particles in the hidden sector. Indeed the particles $X^{\prime}$ can have a very small electric charge, which may arise naturally in a unified 
non-Abelian gauge theory [22], so that the Compton scattering reaction $\gamma X^{\prime} \rightarrow \gamma^{\prime} X^{\prime}$ shown in Figure 1 can recouple the two sectors.

The rate of this process may be estimated as follows. Wave function mixing mediated through a one-loop diagram involving the connector particles generates a term $(\epsilon / 2) F^{\mu \nu} F_{\mu \nu}^{\prime}$ in the Lagrangian, where $\epsilon \simeq\left(e e^{\prime} / 16 \pi^{2}\right) v v^{\prime} / M_{\mathrm{C}}^{2}$ for $M_{\mathrm{C}} \gg v, v^{\prime}$ and values of $\epsilon \lesssim 10^{-3}$ may be considered natural [20]. After diagonalizing the kinetic terms, the interactions of the photons can be written in several alternative bases. Let $J^{\mu}$ and $J^{\prime \mu}$ be the currents, including the gauge coupling constants $e$ and $e^{\prime}$, for $\mathrm{U}(1)$ and $\mathrm{U}(1)^{\prime}$ respectively. When only ordinary matter is relevant it is most convenient to write the corresponding Lagrangian to order $\epsilon$ in the form

$$
\mathcal{L}_{\mathrm{int}}=a^{\mu} J_{\mu}+\left(a^{\prime \mu}+\epsilon a^{\mu}\right) J_{\mu}^{\prime},
$$

where $a^{\mu}$ is defined to be the field coupled to $J^{\mu}$ which creates "our" photon $\gamma$. (Note that the quanta coupled to $J^{\mu}$ and $J^{\prime \mu}$ are not orthogonal. Thus the field $a^{\prime \mu}$ does not create the shadow photon $\gamma^{\prime}$ of the hidden sector; this is done instead by a field $A^{\prime \mu}$.) From eq.(3) we see that the hidden particles $X^{\prime}$ interact with the photon through a very small electromagnetic charge $\epsilon e^{\prime}$. For fermionic $X^{\prime}$ species the rate of the recoupling process is then 20]

$$
n_{X^{\prime}}\langle\sigma v\rangle=C n_{X^{\prime}} \frac{\pi \alpha^{\prime 2} \epsilon^{2}}{T T^{\prime}}
$$

where $\alpha^{\prime}=e^{\prime 2} / 4 \pi$ and $C$ is a number of $\mathcal{O}(1)$ which comes from thermally averaging over the energy transfer. As we shall see, constraints from the $\mathrm{CMB}$ require the recoupling reaction to occur during the radiation-dominated era. The energy density of the universe is then dominated by the plasma in the visible sector. Defining the recoupling temperature $T_{\mathrm{R}}$ to be the temperature of our plasma when the recoupling rate equals the expansion rate gives [20]

$$
\tilde{\alpha} \simeq 10^{-22} \frac{1}{C}\left(\frac{T}{T^{\prime}}\right)_{\mathrm{R}}^{2}\left(\frac{T_{\mathrm{R}}}{10 \mathrm{keV}}\right)\left(\frac{0.1}{\alpha^{\prime}}\right)
$$

where $\tilde{\alpha} \equiv \epsilon^{2} \alpha^{\prime} \sum_{i} g_{i}^{\prime} q_{i}^{2}$ and $g_{i}^{\prime}$ is the number of spin states (assumed fermionic) of the hidden plasma having interaction $q_{i} e^{\prime}$ with $\gamma^{\prime}$. To calculate the resultant cooling of the visible sector it is assumed that the energy transfer occurs quickly compared with the expansion rate; the final temperature $T_{\mathrm{F}}$ is then obtained by demanding energy conservation at recoupling, i.e. $g T_{\mathrm{R}}^{4}+g^{\prime} T_{\mathrm{R}}^{\prime 4}=\left(g+g^{\prime}\right) T_{\mathrm{F}}^{4}$. The energy density of the shadow sector should be negligible during BBN (in order not to increase the expansion rate excessively) but dominates after recoupling (because $g^{\prime} \gg g=2$ ) which gives $n_{\gamma}\left(T_{\mathrm{F}}\right) / n_{\gamma}\left(T_{\mathrm{R}}\right) \simeq\left(g / g^{\prime}\right)^{3 / 4}$. Therefore a critical density nucleonic universe (see eq.(2)) requires $g^{\prime} \sim 40-1000$ and, correspondingly, $T / T^{\prime} \gtrsim 2-5$. Subsequently the $X^{\prime}$ particles acquire mass through a phase transition with a critical temperature bounded as

$$
T_{\mathrm{C}}^{\prime}<T_{\mathrm{R}}^{\prime}<T_{\mathrm{R}} / 2
$$

It would be natural to expect that $m_{X^{\prime}}$ is of $\mathcal{O}\left(T_{\mathrm{C}}^{\prime}\right)$.

\section{A. Constraint from BBN}

Nucleosynthesis obviously provides an upper bound on the recoupling temperature since the abundances of the elements would be altered if it is too high. To study this, we have modified the 
standard computer code [23] to include an abrupt drop in temperature at $T_{\mathrm{R}}$ when the recoupling reaction occurs. Before this, the universe contains two plasmas which are not in thermal contact, the usual one characterized by the photon temperature $T \equiv T_{\gamma}$ (consisting of $\gamma, 3 \nu^{\prime}$ s, $e^{ \pm}$, as well as nucleons), and the other one containing the shadow sector particles like $\gamma^{\prime}$ and $X^{\prime}$ with temperature $T^{\prime}$. An important parameter is the ratio of the shadow sector temperature to the neutrino temperature before recoupling,

$$
r \equiv \frac{T^{\prime}}{T_{\nu}}
$$

which stays constant during $e^{+} e^{-}$annihilation when the photon temperature $T$ increases (but not $T^{\prime}$ or $T_{\nu}$ ). It enables the energy density $\rho^{\prime}$ of the shadow sector before recoupling to be tracked using the neutrino energy density, $\rho^{\prime}=\rho_{\nu} g^{\prime} r^{4} / g_{\nu}$, and is thus a more convenient parameter than $T^{\prime} / T$ as used in ref. 20]. Now at the recoupling temperature $T_{\mathrm{R}}$ there is a sudden drop (which would be smooth in a more detailed model) to the final temperature $T_{\mathrm{F}}$. Energy conservation gives

$$
\frac{\pi^{2}}{30}\left(g_{\gamma}+g_{\nu}+g_{\mathrm{e}}\right) T_{\mathrm{R}}^{4}+\rho^{\prime}\left(T_{\mathrm{R}}^{\prime}\right)=\frac{\pi^{2}}{30}\left(g_{\gamma}+g_{\nu}+g_{\mathrm{e}}\right) T_{\mathrm{F}}^{4}+\frac{\pi^{2}}{30} g^{\prime} T_{\mathrm{F}}^{4},
$$

where $g_{\gamma}=2, g_{\nu}=21 / 4$ and $g_{\mathrm{e}}=7 / 2$. Since $T_{\mathrm{R}}=T_{\mathrm{F}}$ for neutrinos, the corresponding terms above cancel and we obtain (neglecting the electron energy density for the temperatures of relevance),

$$
T_{\mathrm{F}}=A T_{\mathrm{R}}, \quad A \equiv\left[\frac{2+\left(\frac{4}{11}\right)^{4 / 3} g^{\prime} r^{4}}{2+g^{\prime}}\right]^{1 / 4}
$$

After recoupling the energy density of the shadow sector is just $\rho^{\prime}=\frac{\pi^{2}}{30} g^{\prime} T^{4}$ since now $T=T^{\prime}$.

We have implemented a routine in the BBN code dividing the thermal history into the three steps (before, during and after recoupling) and compute the expansion rate accordingly, taking into account the contribution of shadow particles. We assume 3 light neutrino species, use the neutron lifetime $\tau_{n}=887 \pm 2 \mathrm{~s}$ [16], and incorporate small corrections to the helium abundance as reviewed in ref. [13]. Since the main effect of photon cooling is to increase the helium-4 abundance and decrease the deuterium abundance, we bound their values from recent observations of helium in metal-poor extragalactic HII regions [24] and of deuterium in the interstellar medium [25]:

$$
Y_{\mathrm{P}}\left({ }^{4} \mathrm{He}\right) \leq 0.25, \quad(\mathrm{D} / \mathrm{H})_{\mathrm{p}} \geq 1.1 \times 10^{-5} .
$$

We do not consider the bound on ${ }^{7} \mathrm{Li}$ because it does not provide a useful constraint. The adopted bounds are very conservative, based upon consideration of a variety of data which are critically discussed elsewhere 13]. As shown in Figure 2, we can then derive the maximum value of $T_{\mathrm{R}}$ allowed for each given set of parameters $r$ and $g^{\prime}$. The allowed region is:

$$
T_{\mathrm{R}}<74 \mathrm{keV}, \quad r<0.3 \quad \text { and } \quad 40<g^{\prime}<2340,
$$

only part of which is shown in the figure for convenience. (The highest allowed value of $g^{\prime}$ corresponds to the smallest initial value of $\eta$, viz. $\eta_{\mathrm{BBN}}>1.7 \times 10^{-10}$ [3].) For comparison, the values suggested in ref. [20] were $T_{\mathrm{R}} \lesssim 30 \mathrm{keV}, r \lesssim 0.7$ and $g^{\prime} \sim 40-1000$. 


\section{B. Constraint from the CMB spectrum}

A lower bound on the recoupling temperature follows from the observation by COBE that the CMB spectrum is accurately blackbody [21]. The recoupling reaction removes photons from the thermal bath thus inducing a chemical potential for the photon distribution. If the reaction occurs early enough, however, photon creation processes can restore the Planck spectrum [26] in agreement with the observational upper limit [21] on such a chemical potential: $\mu<9 \times 10^{-5}(95 \%$ c.l.). To thus constrain $T_{\mathrm{R}}$ we use a simple argument concerning the relevant time scales.

At temperatures below the $e^{+} e^{-}$threshold, the processes which determine the spectral shape are Compton scattering (C) which preserves the photon number, and the radiative processes bremsstrahlung (B) and double Compton scattering (DC). The time scales of these processes are

$$
\begin{aligned}
t_{\mathrm{C}} & =9.25 \times 10^{-7} \sec \frac{1}{\eta}\left(\frac{T}{\mathrm{keV}}\right)^{-4}, \\
t_{\mathrm{DC}} & =2.53 \times 10^{-2} \sec \frac{1}{\eta}\left(\frac{T}{\mathrm{keV}}\right)^{-5}, \\
t_{\mathrm{B}} & =2.18 \times 10^{-8} \sec \frac{1}{\eta^{2}}\left(\frac{T}{\mathrm{keV}}\right)^{-5 / 2} \ln ^{-2}\left[2.13 \frac{1}{\sqrt{\eta}}\left(\frac{T}{\mathrm{keV}}\right)^{3 / 4}\right] .
\end{aligned}
$$

For a $\Omega_{\mathrm{N}}=1$ universe, the value of $\eta$ after recoupling (i.e. today) is given by eq.(2) whereas before recoupling it is

$$
\eta\left(T \geq T_{\mathrm{R}}\right)=\eta_{0} \frac{n_{\gamma}\left(T_{\mathrm{F}}\right)}{n_{\gamma}\left(T_{\mathrm{R}}\right)}=\eta_{0} A^{3}
$$

where $A$ is defined in eq.(9). (Note that baryons and nucleons are equivalent with regard to the CMB since only their electromagnetic interactions are relevant; therefore we henceforth refer to $\Omega_{\mathrm{B}}$ rather than $\Omega_{\mathrm{N}}$.) The time scales above are to be compared with the age of the universe obtained by integrating the Friedmann equation,

$$
t= \begin{cases}2.42 \times 10^{6} \sec g_{\mathrm{i}}^{-1 / 2}\left(\frac{T}{\mathrm{keV}}\right)^{-2} & \text { for } T>T_{\mathrm{R}} \\ 2.42 \times 10^{6} \mathrm{sec}\left[\left(\frac{1}{\sqrt{g_{\mathrm{i}}}}-\frac{1}{A^{2} \sqrt{g_{\mathrm{F}}}}\right)\left(\frac{T_{\mathrm{R}}}{\mathrm{keV}}\right)^{-2}+\frac{1}{\sqrt{g_{\mathrm{F}}}}\left(\frac{T}{\mathrm{keV}}\right)^{-2}\right] & \text { for } T \leq T_{\mathrm{F}}\end{cases}
$$

where we have used

$$
\begin{aligned}
& g_{\mathrm{i}} \equiv g\left(T>T_{\mathrm{R}}\right)=2+\frac{21}{4}\left(\frac{4}{11}\right)^{4 / 3}+g^{\prime} r^{4}\left(\frac{4}{11}\right)^{4 / 3}, \\
& g_{\mathrm{F}} \equiv g\left(T \leq T_{\mathrm{F}}\right)=2+\frac{21}{4} A^{-4}\left(\frac{4}{11}\right)^{4 / 3}+g^{\prime} .
\end{aligned}
$$

Obviously the abrupt transition at $T_{\mathrm{R}}$ would be smoothed out in a more detailed picture of the phase transition.

In Figure 3 we show the various time scales for a typical set of parameters, with a sudden jump in $\eta$ as the temperature drops from $T_{\mathrm{R}}$ to $T_{\mathrm{F}}$ as determined by eq.(9). Although bremsstrahlung usually dominates at low redshifts for a high density baryonic universe, the most efficient photon

\footnotetext{
${ }^{2}$ We have inserted the correction factor of $4 \pi$ [26] into the rate for bremsstrahlung [27].
} 
creation process is always found to be double Compton scattering for the values of $T_{\mathrm{R}}, r$ and $g^{\prime}$ under consideration. The limiting value of the recoupling temperature is then conservatively obtained by demanding that the time scale for this be shorter than the expansion time scale. We have indicated in the figure the error bands for the time scales in eq.(12) corresponding to the allowed region of $\eta$ after nucleosynthesis, viz. $\eta \in(1.7-8.9) \times 10^{-10}$ [3]. To obtain the minimum recoupling temperature we took the highest possible value for $\eta_{0}$ from eq.(2) and searched for the temperature at which $t \geq t_{\mathrm{DC}}$ is satisfied as a function of the parameters $r$ and $g^{\prime}$; this gives

$$
T_{\mathrm{R}} \geq 0.73 \mathrm{keV}\left(\frac{\sqrt{g_{\mathrm{i}}}}{A^{5}}\right)^{1 / 3} .
$$

Combining the constraints from BBN and the CMB spectrum it follows that the recoupling temperature must be in the range

$$
3 \mathrm{keV}<T_{\mathrm{R}}<75 \mathrm{keV}
$$

for the allowed range of $r$ and $g^{\prime}$ as given in eq.(11).

\section{Constraints from stellar evolution}

Further constraints on the model follow from the energy loss argument for stars [20]. For example if the $X^{\prime}$ particles are light enough to be produced in red giants through plasmon decays, a limit on $\tilde{\alpha}$ (eq.(5)) follows from requiring that the energy loss through $X^{\prime}$ emission does not affect the stellar luminosity or age to a noticeable degree [28]. The resultant constraint is very stringent, viz. $\tilde{\alpha} \lesssim 10^{-28}$ [20], and in conflict with the required value from eq.(5) unless the recoupling temperature is very low. This, however, is not allowed by the CMB spectrum constraint (eq.(16)) so the only escape route is to argue that the $X^{\prime}$ particles are too heavy to be produced in stars [20]. In the cores of red giants the plasmon mass is $\omega_{\mathrm{pl}} \sim 20 \mathrm{keV}$, so this requires $m_{X^{\prime}}>\omega_{\mathrm{pl}} / 2 \gtrsim 10 \mathrm{keV}$. There are additional contributions to $X^{\prime}$ production from Compton and bremsstrahlung processes and to suppress these one would require $m_{X^{\prime}} \gtrsim 5 T$ where $T \sim 10 \mathrm{keV}$ is the core temperature [20]. . $^{3}$ However, if $m_{X^{\prime}}$ is indeed of $\mathcal{O}\left(T_{\mathrm{R}}\right)$ then this implies $T_{\mathrm{R}} \gtrsim 50 \mathrm{keV}$ (see eq.(6)), which exceeds the upper limit from BBN over most of the parameter space (see Figure 2). Thus it would be necessary to invoke some fine-tuning and argue that $m_{X^{\prime}} \gg T_{R}$.

An even more restrictive constraint on $\tilde{\alpha}$ comes from supernova physics. After collapse, the supernova core forms a neutron star which radiates its binding energy primarily in the form of neutrinos which diffuse out of the dense central region over a time scale of seconds. Observations of (anti)neutrinos from SN1987A 29] confirmed that these indeed carried away $\sim 10^{53}$ erg on the expected time scale. A competing mechanism for cooling the core would be the production of $X^{\prime}$ particles through plasmon decays. Then a conservative requirement is that the $X^{\prime}$ particles do not carry away more energy than the neutrinos and thus curtail the duration and energetics of the neutrino burst. Assuming the core radius to be $\sim 10 \mathrm{~km}$ and a cooling time of $\sim 10 \mathrm{~s}$ one obtains for the energy loss $E^{\prime}$ through $X^{\prime}$ [20]

\footnotetext{
${ }^{3}$ Note that processes of the form $e^{-} \gamma \rightarrow e^{-} \gamma^{\prime}$ or $e^{-4} \mathrm{He} \rightarrow e^{-4} \mathrm{He} \gamma^{\prime}$ are not allowed by the Lagrangian of eq.(3); if these existed, the associated energy loss could not be evaded by increasing $m_{X^{\prime}}$.
} 


$$
E^{\prime} \approx 10^{71} \operatorname{erg} \tilde{\alpha} \rho_{14}^{2 / 3} T_{10}^{3} \lesssim 10^{53} \operatorname{erg}
$$

where $T_{10} \equiv T /(10 \mathrm{MeV}), \rho_{14} \equiv \rho /\left(10^{14} \mathrm{~g} / \mathrm{cm}^{3}\right)$, and the $X^{\prime}$ particles are assumed to be lighter than the plasmon mass in the supernova core. Typical values for the density and temperature are $\rho_{14} \sim 6$ and $T_{10} \sim 5$ [30]. Solving this equation for $\tilde{\alpha}$ and inserting into eq.(5) we then find:

$$
T_{\mathrm{R}} \lesssim 3.6 \times 10^{5} \mathrm{keV} \rho_{14}^{-2 / 3} T_{10}^{-3} C \alpha^{\prime} r^{2}
$$

In Figure 1 we show that this constraint together with those from BBN and the CMB spectrum effectively rules out the model (taking $C=1$, and $\alpha^{\prime}=0.1$ ). The only allowed region is a tiny corner at the highest value of $r$ and the lowest value of $g^{\prime}$, fixing the recoupling temperature to be $7-8 \mathrm{keV}$. This region is further limited by decreasing $\alpha^{\prime}$ or $C$. Of course the physical parameters of supernova collapse are somewhat uncertain so this argument is not definitive. One can only conclude that $X^{\prime}$ particles, if they exist, may play an essential role in the supernova process [20].

\section{Constraints from LSS and CMB anisotropy}

Finally we consider the formation of large scale structure and the concomitant generation of small scale CMB anisotropy in a critical density baryonic universe.t In the standard picture, such structure grows by gravitational instability from a (nearly) scale-invariant spectrum of primordial adiabatic, gaussian fluctuations (with power spectrum $P(k) \propto k^{n}, n \approx 1$ ), presumably generated during an inflationary phase in the early universe [31. On scales smaller than the horizon size at matter-radiation equality the primordial spectrum is modified and its slope becomes negative. The COBE detection of anisotropy in the CMB on angular scales exceeding the horizon size at the recombination epoch [32] has provided strong support for this basic picture and moreover fixed the normalization of the primordial spectrum. Subsequent observations of anisotropy on small angular scales have also provided evidence for a 'Doppler peak' in the angular power spectrum such as would be expected from acoustic oscillations of the coupled plasma and photon fluids during the last scattering of the CMB [31]. Besides the observed power spectrum of galaxy clustering, we thus have a new quantitative test for models of structure formation.

A baryonic universe was of course the first to be investigated in the context of structure formation [33]. The salient feature of such a universe is that fluctuations can begin to grow only after (re)combination (rather than as soon as the universe becomes matter-dominated) since the baryons are tightly coupled to the photons before this epoch. Moreover the coupling is not perfect (and becomes weaker as the universe turns neutral) so adiabatic fluctuations suffer 'Silk damping' on scales smaller than the damping mass of $M_{\mathrm{D}} \approx 1.3 \times 10^{12}\left(\Omega_{\mathrm{B}} h^{2}\right)^{-3 / 2} M_{\odot}$. It was recognized early on that primordial gaussian fluctuations with spectral index $n \lesssim 2$ would thus result in excessively large fluctuations in the mass distribution on small scales [34]. Moreover the upper bounds on CMB anisotropy available a decade ago already implied that there would not be sufficient time after

\footnotetext{
${ }^{4}$ Note that in the Bartlett-Hall model [20] the $X^{\prime}$ particles of the shadow sector can be a candidate for the dark matter. Since $n_{X^{\prime}} \sim n_{\gamma}$ at recoupling, there will be a large contribution to the density parameter $\left(\Omega_{X^{\prime}} \sim 40 h^{-2}\left(m_{X^{\prime}} / \mathrm{keV}\right)\right)$ unless the $X^{\prime}$ particles subsequently self-annihilate efficiently after becoming non-relativistic. This is however quite possible and, in fact, very likely in analogy to the 'visible' sector.
} 
(re)combination for the implied small primordial fluctuations to grow to the large-scale structure observed today in a baryonic universe [35].

In the past particle physicists have tended to ignore such constraints since there did not exist a 'Standard Model' for the formation of structure. The increasing weight of observational evidence for the picture outlined above, however, now makes such a viewpoint untenable. In particular the data (as opposed to upper limits) on $\mathrm{CMB}$ anisotropy now allow a precise quantitative test of a baryon-dominated universe. For a $\Omega_{\mathrm{B}}=1$ universe, the slope of the primordial spectrum $n$ and the Hubble parameter $h$ are the only free parameters. Thus we will first determine the values of $n$ and $h$ which would allow a fit to the data on small-scale CMB anisotropy. These are available as measurements of the multipole moments $C_{l}=\frac{1}{2 l+1} \sum_{m} a_{l m}^{2}$, expanding the CMB temperature on the celestial sphere in spherical harmonics:

$$
\Delta T=\sum_{l, m} a_{l m} Y_{l m}(\theta, \phi)
$$

Then we can check if the power spectrum of galaxy clustering can be reproduced for the same choice of parameters. We emphasize that this test is thus as model-independent as possible.

To compute the angular power spectrum for each model specified by a pair $(n, h)$, we use a fast Boltzmann code [36] and adopt the COBE-normalization at large angular scales. Then we convolve each power spectrum with the experimental 'window functions' before comparison with the observational data by generating a $\chi^{2}$ surface over the $(n, h)$ space under investigation; this method is outlined in detail in ref. [37]. The $\chi^{2}$ value is computed according to

$$
\chi^{2}(n, h)=\sum_{N=1}^{N_{\text {exp }}}\left[\frac{\delta T_{l_{\text {eff }}^{\text {data }}}(N)-\delta T_{l_{\text {eff }}^{\text {model }}(N, n, h)}}{\sigma^{\text {data }}(N)}\right]^{2},
$$

where the sum is over all the CMB observations, $\delta T_{l_{\text {eff }}}^{\text {model }}(N, n, h)$ and $\delta T_{l_{\mathrm{eff}}}^{\text {data }}(N)$ are, respectively, the flat band powers (i.e. convolved with the experiment-specific 'window functions') for the models and observations, and $\sigma^{\text {data }}(N)$ are the associated observational errors. The CMB data we consider and the corresponding errors are tabulated in [37] and shown in Figure : 5 . (We consider only the central value of the Saskatoon data which have a $\pm 14 \%$ calibration uncertainty.) The band powers are given by

$$
\delta T_{l_{\mathrm{eff}}^{\mathrm{model}}}=\left[\frac{1}{I\left(W_{l}(N)\right)} \sum_{l=2}^{l_{\max }} \frac{2 l+1}{4 \pi} C_{l}(n, h) W_{l}(N)\right]^{1 / 2}
$$

where the deconvolving factors $I\left(W_{l}(N)\right)$ are the logarithmic integrals of the experimental window functions $W_{l}(N)$ defined as

$$
I\left(W_{l}\right)=2 \pi \sum_{l=2}^{l_{\max }} \frac{(2 l+1) W_{l}}{4 \pi l(l+1)},
$$

and $l_{\max }$ can be safely taken to be 1200 for all existing data (for larger $l$ the acceptance is negligible). Finally, the angular scale investigated by experiment $N$ is given according to

$$
l_{\mathrm{eff}}(N)=\frac{I\left(l W_{l}(N)\right)}{I\left(W_{l}(N)\right)} .
$$


To obtain a $\chi^{2}$ value for each point of the $(n, h)$ space we use the data and eq.(22) for each observation $N$ and sum over all existing observations. The values of the multipoles $C_{l}(n, h)$ are obtained by running the fast Boltzmann code mentioned above.

In Figure 6 we show the region of $(n, h)$ space which is consistent with the data. We investigate the parameter range $0.25 \leq h \leq 1$ and $0.5 \leq n \leq 1.5$, the former covering all direct measurements [16] and the latter based on general theoretical considerations of inflationary models [38]. We show both the $68 \%$ C.L. and $95 \%$ C.L. contours; the latter delineate the range

$$
0.59 \leq n \leq 0.78, \quad 0.41 \leq h \leq 0.62
$$

The negative correlation of $n$ and $h$ arises because the amplitude of the acoustic peak plays the dominant role in the fitting procedure and for $\Omega_{\mathrm{B}}=1$ this peak amplitude decreases as $h$ and $n$ decrease. Thus a decrease in $n$ accompanied by a correlated increase in $h$ (or vice versa) can preserve the amplitude and thus the $\chi^{2}$ value for the fit. In general rather low values of both $n$ and $h$ are required to fit the acoustic peak in a critical density baryonic universe.

Given these favoured values of $n$ and $h$ we can now calculate the linear matter power spectrum. In Figure 7 we show the spectra for the two extreme models in the $(n, h)$ plane, viz. $n=0.5$, $h=0.62$ and $n=0.78, h=0.25$, along with the data points inferred from the APM galaxy survey [39]. To compute these spectra we have used the COSMICS code 40] and normalized to COBE using the prescription given in ref. 41. The strong oscillations seen at short scales arise from matter fluctuations on wavelengths smaller than the Hubble radius at (re)combination and reflect the value of the phase of the perturbation at recombination [34]. It is seen that even for extreme choices of parameters, the power spectra of $\Omega_{\mathrm{B}}=1$ models disagree strongly with the observational data. Even though the clustering of APM galaxies may be enhanced over that of the matter distribution, the discrepancy cannot be resolved for any sensible value of the 'bias' parameter. The situation is further aggravated if the value of $h$ is decreased in Figure $\mathbb{Z}(\mathrm{a})$ or the value of $n$ is decreased in Figure 7(b), staying within the allowed region of eq.(25), since in both cases the matter power spectrum is further damped at short scales. Thus we can firmly establish that it is not possible to simultaneously satisfy the LSS and CMB data in an universe with the critical density in baryons.

\section{DISCUSSION}

Although big bang nucleosynthesis is usually cited as providing the main argument for nonbaryonic dark matter, it is possible to entertain deviations from the standard picture in order to accommodate a baryon-dominated critical density universe. One such suggestion invoking a reduction in the comoving entropy after nucleosynthesis [20] is particularly interesting because it challenges the standard assumption that the thermodynamic history of the universe is well known at least up until the BBN epoch. We have demonstrated that this model is very tightly constrained

\footnotetext{
${ }^{5}$ We ignore a possible contribution from gravitational waves (which would lower the COBE normalization) and adopt for the helium fraction the central value $Y_{\mathrm{P}}\left({ }^{4} \mathrm{He}\right)=0.24$ 24]. Note also that the error bars $\sigma^{\text {data }}(N)$ are mostly asymmetric; to accommodate this in the $\chi^{2}$ calculation, we establish for each data point whether the model lies above or below and then take the appropriate error bar in eq.(22).
} 
(if not ruled out altogether) from consideration of other processes such as thermalization of the CMB spectrum and the effects on stellar evolution of the new physics invoked by the model. Finally we have shown that recent observations of small-scale CMB anisotropy and large-scale structure decisively rule out this model. This conclusion also holds for other models which attempt to evade the constraint from nucleosynthesis in order to allow a baryonic Einstein-De Sitter universe.

Acknowledgments. We would like to thank Charles Lineweaver for supplying the window functions for CMB experiments, Uros Seljak and Matias Zaldarriaga for providing the Boltzmann code, and Leith Cooper, Lawrence Hall, Georg Raffelt and Matias Zaldarriaga for helpful discussions. M.B. gratefully acknowledges financial support from the Fellowship HSP II/AUFE of the German Academic Exchange Service (DAAD). This work was supported by the EC Theoretical Astroparticle Network CHRX-CT93-0120 (DG12 COMA). 


\section{REFERENCES}

[1] P.J.E. Peebles, Physical Cosmology (Princeton Univ. Press, 1993).

[2] H. Reeves et al, Astrophys. J. 179 (1973) 909.

[3] P.J. Kernan and S. Sarkar, Phys. Rev. D54 (1996) R3681.

[4] E. Witten, Phys.Rev. D30 (1984) 272.

[5] J. Alam, S. Raha and B. Sinha, astro-ph/9704226.

[6] J. Ellis, Nuovo Cimento A107 (1994) 1091.

[7] B.J. Carr, Ann. Rev. Astron. Astrophys. 32 (1994) 531.

[8] C. Alcock et al (MACHO collab.), Astrophys. J. 461 (1996) 84, 471 (1996) 774.

[9] J.-M. Alimi and A. Serna, astro-ph/9704254.

[10] G.D. Starkman, Phys. Rev. D45 (1992) 476.

[11] J.H. Applegate, C. Hogan and E.J. Scherrer, Astrophys. J. 329 (1988) 572.

[12] R.A. Malaney and G.J. Mathews, Phys. Rep. 229 (1993) 145.

[13] S. Sarkar, Rep. Prog. Phys. 59 (1996) 1493.

[14] G. Gyuk and M.S. Turner, Phys. Rev. D50 (1994) 6130.

[15] S. Sarkar and A.M. Cooper, Phys. Lett. 148B (1984) 347.

[16] Particle Data Group, Phys. Rev. D54 (1996) 1.

[17] S. Dimopoulos et al, Astrophys. J. 330 (1988) 545.

[18] G. Steigman et al, Astrophys. J. 415 (1993) L35.

[19] E.D. Carlson et al, Phys. Rev. Lett. 65 (1990) 2225.

[20] J.G. Bartlett and L.J. Hall, Phys. Rev. Lett. 66 (1991) 541.

[21] D.J. Fixen et al (COBE collab.), Astrophys. J. 473 (1996) 576.

[22] B. Holdom, Phys. Lett. B166 (1986) 196.

[23] R.V. Wagoner, Astrophys. J. 179 (1973) 343;

L. Kawano, Preprint FERMILAB-Pub-92/04-A.

[24] Y.I. Izotov, T.X. Thuan and V.A. Lipovetsky, Astrophys. J. Supp. 108 (1997) 1.

[25] P.R. McCullough, Astrophys. J. 390 (1992) 213;

J.L. Linsky et al, Astrophys. J. 402 (1993) 694, 451 (1995) 335.

[26] W. Hu and J. Silk, Phys. Rev. D48 (1993) 485.

[27] A.P. Lightman, Astrophys. J. 244 (1981) 392.

[28] G.G. Raffelt, D. Dearborn and J. Silk, Astrophys. J. 336 (1989) 61.

[29] Y. Totsuka, Rep. Prog. Phys. 55 (1992) 377.

[30] G.G. Raffelt, Stars as Laboratories for Fundamental Physics (Univ. of Chicago Press, 1996).

[31] G. Efstathiou, Physics of the Early Universe, ed. J. Peacock et al (Adam Hilger, 1990) p 361.

[32] C.L. Bennett et al (COBE collab.), Astrophys. J. 464 (1996) L1.

[33] P.J.E. Peebles and J.T. Yu, Astrophys. J. 162 (1970) 815;

M.L. Wilson and J. Silk, Astrophys. J. 243 (1981) 14.

[34] P.J.E. Peebles, Astrophys. J. 248 (1981) 885.

[35] J.R. Bond and G. Efstathiou, Astrophys. J. 285 (1985) L45.

[36] U. Seljak and M. Zaldarriaga, Astrophys. J. 469 (1996) 437.

[37] C.H. Lineweaver et al, astro-ph/9610133.

[38] D.H. Lyth, hep-ph/9609431.

[39] C.M. Baugh and G.P. Efstathiou, Mon. Not. R. Astr. Soc. 265 (1993) 145.

[40] E. Bertschinger, astro-ph/9506070.

[41] E.F. Bunn and M. White, Astrophys. J. 480 (1997) 6. 


\section{FIGURES}

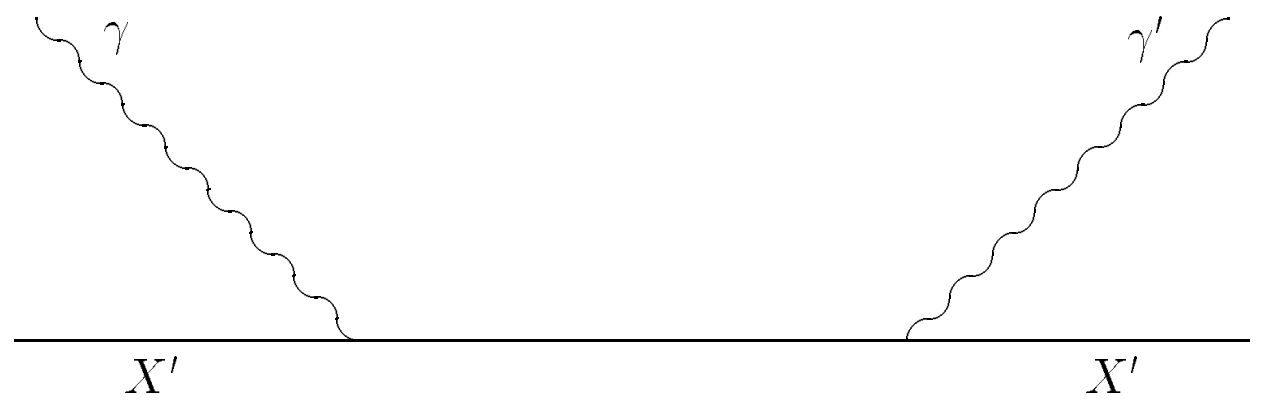

FIG. 1. The Bartlett-Hall mechanism for recoupling of the two sectors via photon mixing.

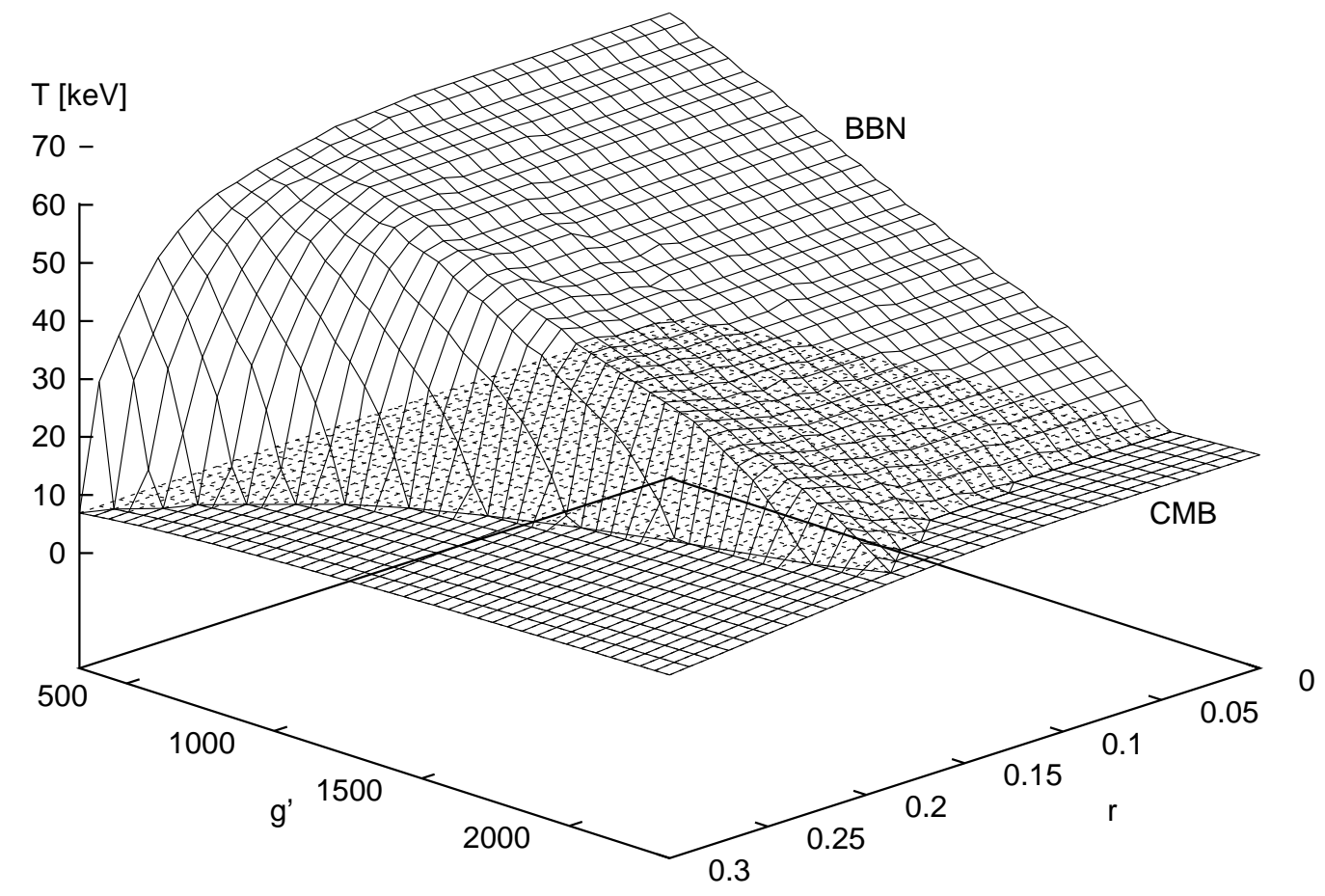

FIG. 2. Constraints on the recoupling temperature as a function of the model parameters $r$ and $g^{\prime}$ from BBN and the CMB spectrum. The allowed region is between the two surfaces. 


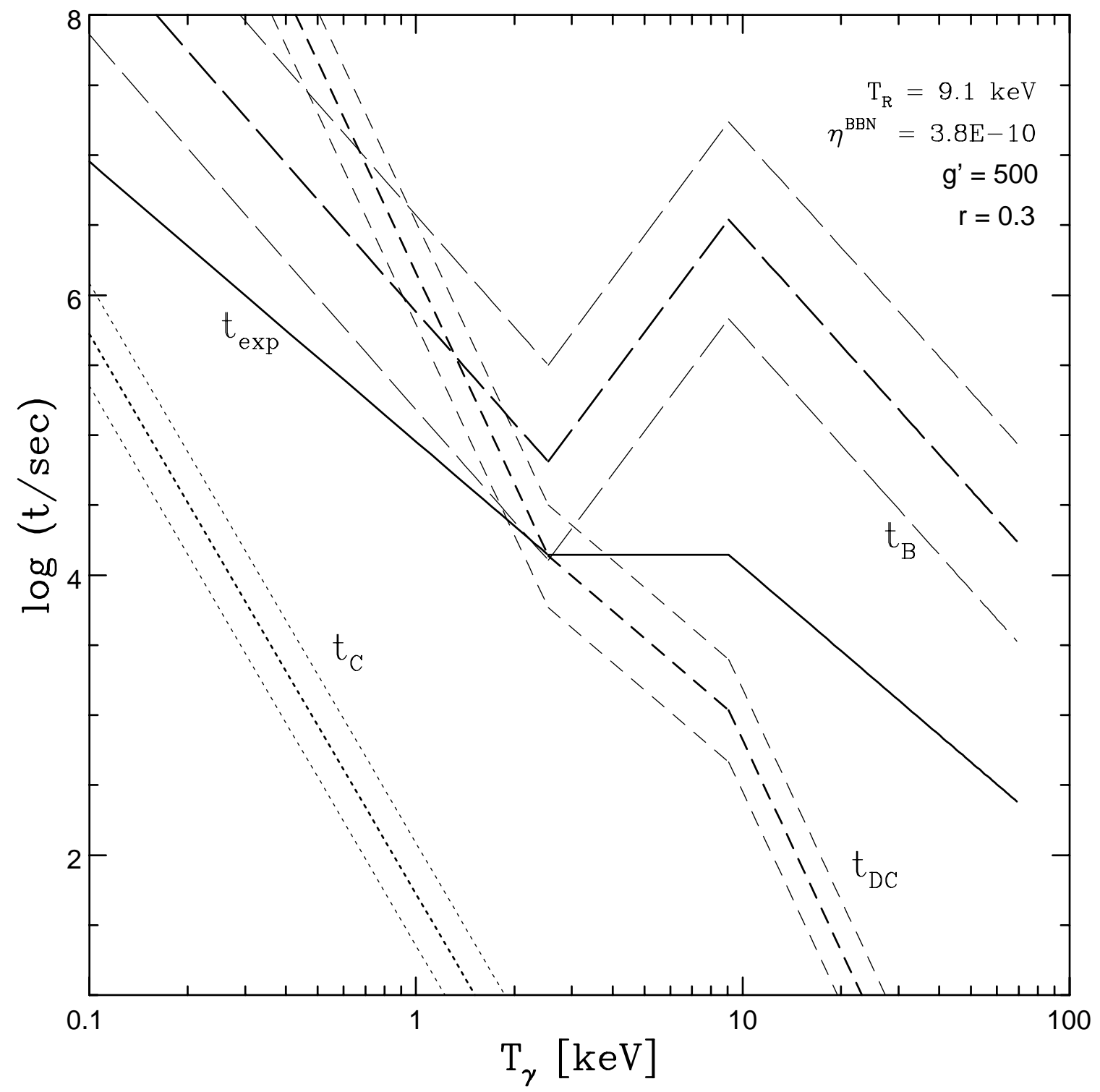

FIG. 3. Time scales for Compton scattering, double Compton scattering and bremsstrahlung compared with the expansion time scale in the Bartlett-Hall model. The steps correspond to the recoupling process at which the temperature drops from $T_{\mathrm{R}}$ to $T_{\mathrm{F}}$ and the comoving photon density decreases by a factor of $\sim 10-100$. 


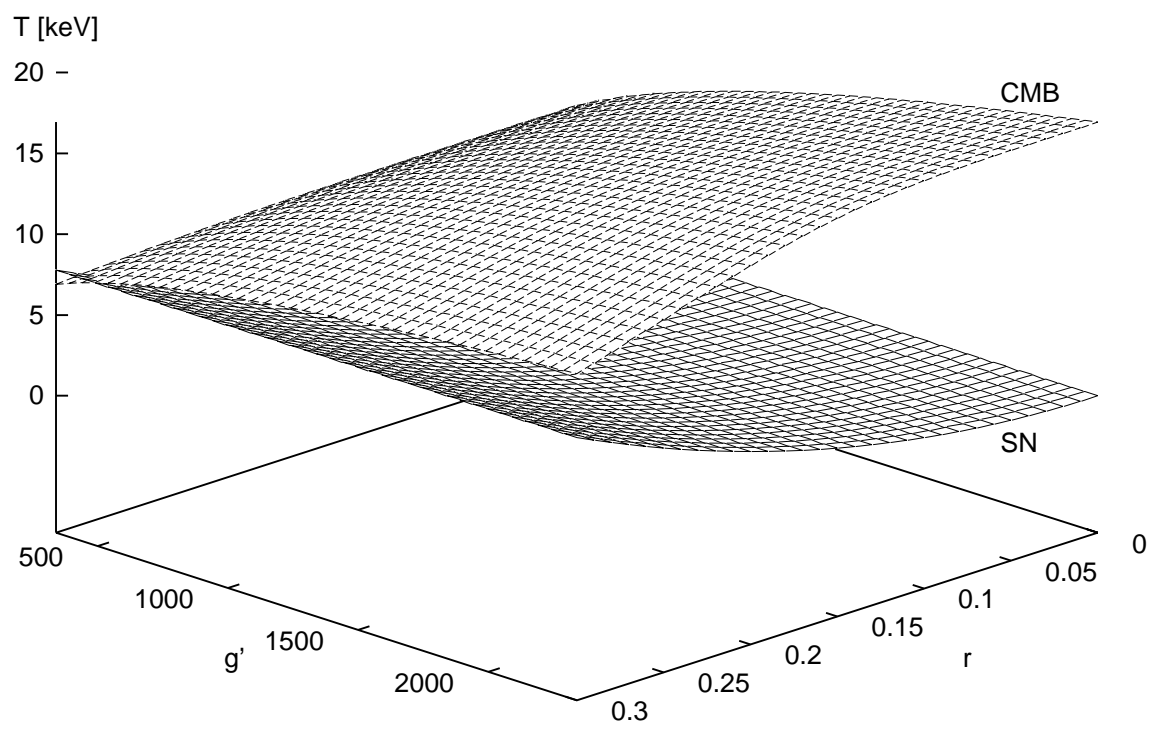

FIG. 4. Constraints on the recoupling temperature as a function of the model parameters $r$ and $g^{\prime}$ from the CMB spectrum and supernova physics (for $\rho_{14}=6, T_{10}=5, C=1$ and $\alpha^{\prime}=0.1$ ). The region between the surfaces is excluded. The only allowed region obtains for the highest values of $r$ and the lowest for $g^{\prime}$, fixing the recoupling temperature to be $\sim 7 \mathrm{keV}$.

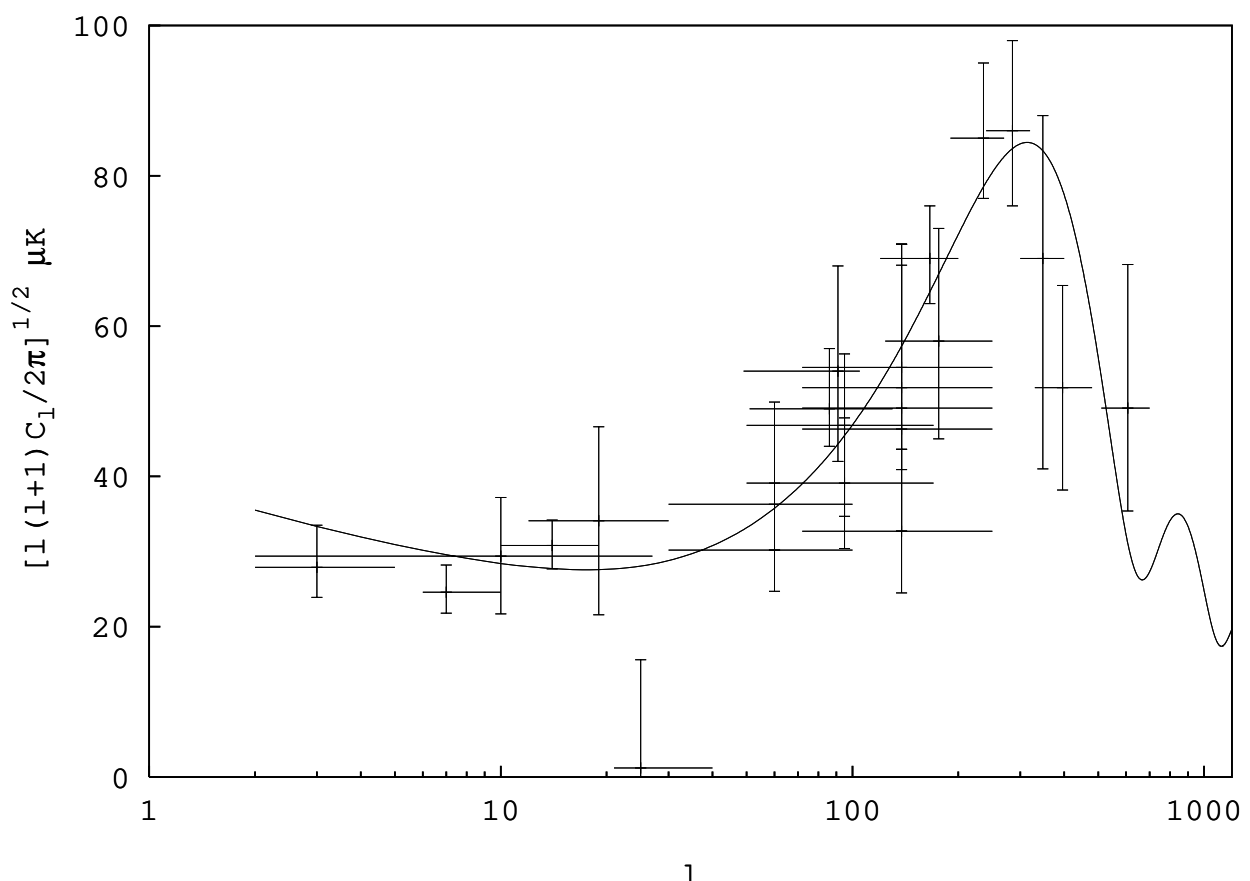

FIG. 5. An illustrative fit to the data on the CMB angular power spectrum of a $\Omega_{\mathrm{B}}=1$ universe with primordial spectrum slope $n=0.66$ and Hubble parameter $h=0.3$ ). 


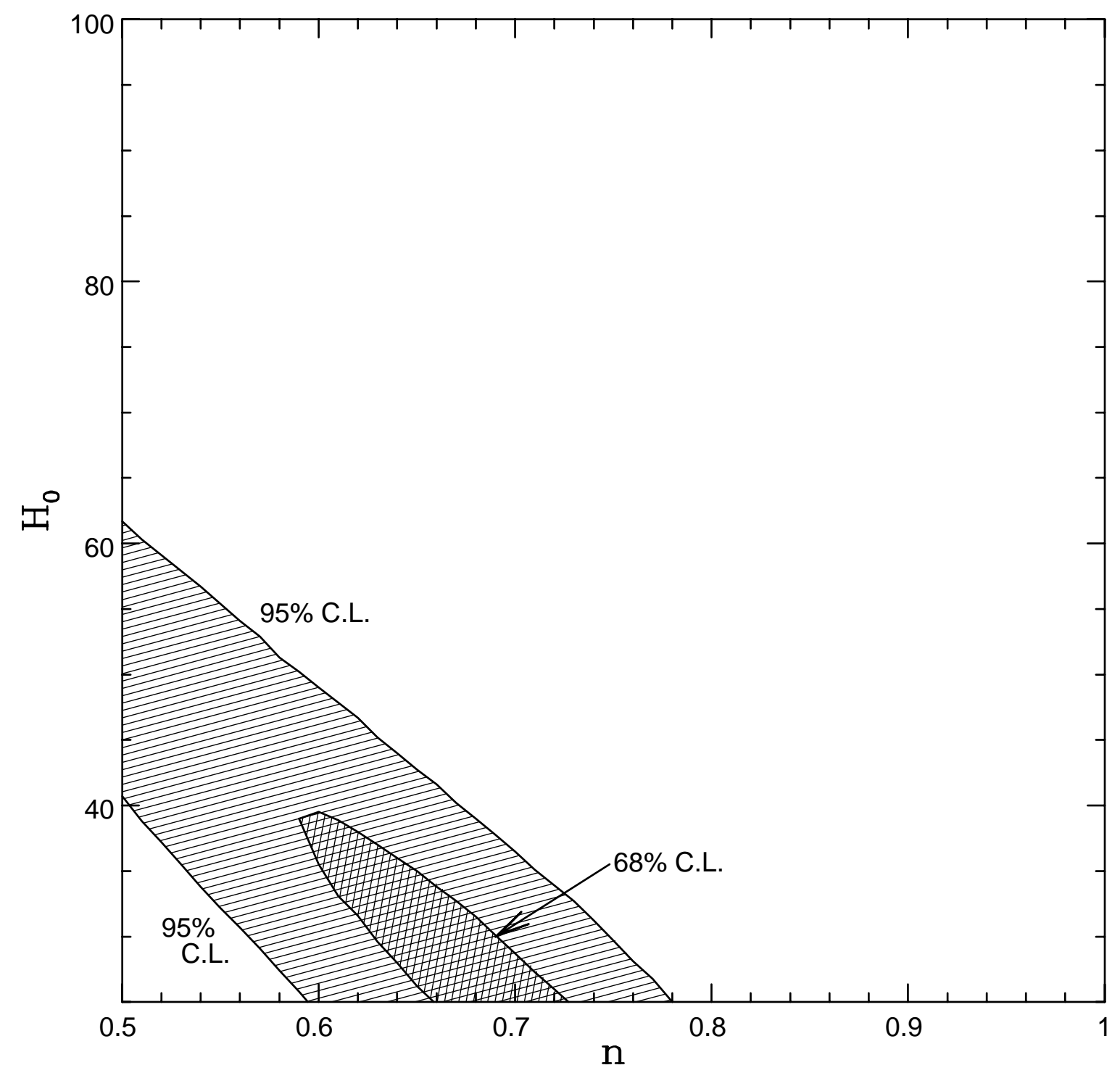

FIG. 6. $\chi^{2}$ contours ( $68 \%$ and $95 \%$ C.L.) in the $n-H_{0}$ plane for the fit to observations of CMB anisotropy of a $\Omega_{\mathrm{B}}=1$ universe. The negative correlation between the primordial spectral index and the Hubble parameter is discussed in the text. 

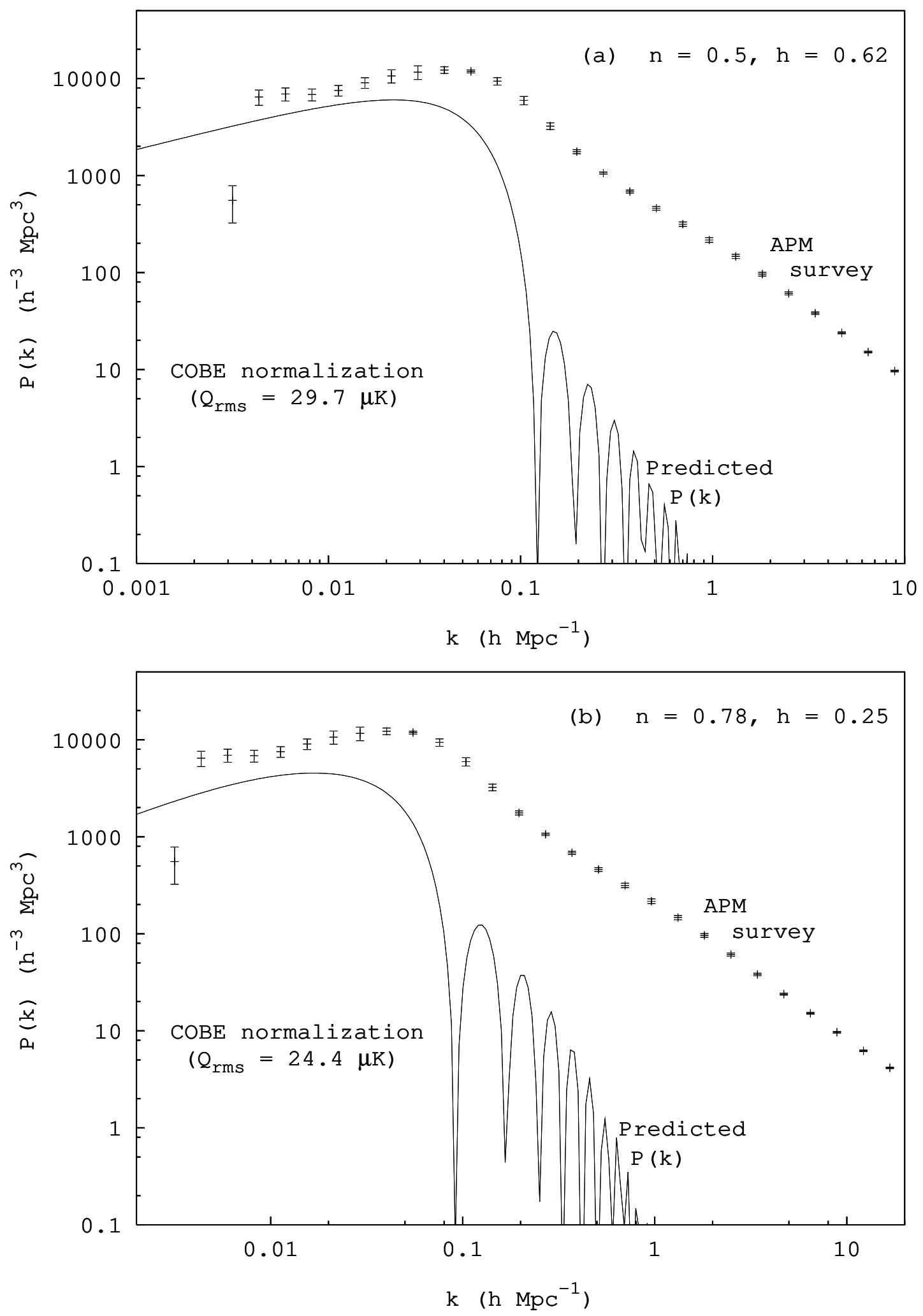

FIG. 7. Predicted power spectra of density fluctuations normalized to COBE for two $\Omega_{\mathrm{B}}=1$ models, (a) $n=0.5, h=0.62$ and (b) $n=0.78, h=0.25$, compared with APM data. 